\title{
RISKS OF CONTRAST MEDIA USE
}

The aim of using CM is to visualize certain structures in the organism or improve their visualization and to obtain information on organ functions. A CM must have low toxicity and be safe to use since it is often injected at a high dose and for certain purposes very quickly.

\section{Barium sulfate}

Barium sulfate is insoluble in water and usually safe when administered orally or rectally for imaging the intestine. While barium ions are toxic, the small amounts present in the suspension of the insoluble barium sulfate and available for intestinal absorption can normally be ignored. Serious complications after barium examinations of the upper and lower GI tracts are few [79]. There are, however, some patients at risk in whom barium sulfate should not be used because of possible aspiration or suspected perforation. Once barium sulfate enters the lungs or leaks from the stomach or intestine, excretion is impossible or very slow and persistent inflammation causes severe problems. Some of the most serious adverse effects after administration of barium preparations are:

- Retention of barium in the colon for some weeks or longer in elderly patients or patients with partial colonic obstruction

- Formation of barium fecoliths, sometimes seen in diverticula of the colon

- Leakage of barium into the peritoneal cavity

- Aspiration of barium into the bronchial tree

- Hypersensitivity reactions to barium preparations, caused by the additives of the barium preparations [80]. 


\begin{tabular}{lrrc} 
Kind of examination & No. of patients & Incidence of side effects (\%) & No. of deaths \\
Urography & 214,033 & $10,257(4.80)$ & 11 \\
\hline Cholagiography & 33,778 & $2,676(8.00)$ & 2 \\
\hline Cerebral angiography & 12,771 & $263(2.06)$ & 1 \\
\hline Angiocardiography & 7,911 & $179(2.26)$ & 2 \\
\hline Aortography & 24,885 & $665(2.67)$ & 1 \\
\hline Other angiographies & 2,815 & $101(3.58)$ & 0 \\
\hline Venography & 5,890 & $160(2.72)$ & 1 \\
\hline Total & $\mathbf{3 0 2 , 0 8 3}$ & $\mathbf{1 4 , 3 0 1 ( 4 . 7 3 )}$ & $\mathbf{1 8 ~ ( 0 . 0 0 6 \% )}$
\end{tabular}

Table. 15. Incidence of side effects in various $\mathrm{CM}$ examinations [81] using ionic CM

\section{Ionic contrast media}

Extensive statistics are available on the side effects of ionic $\mathrm{CM}$. The incidence of reactions depends on a large number of factors, such as the patient's general condition, the kind of examination (table 15), the type of $\mathrm{CM}$, its dose, and also the conditions under which the examination is being conducted. Special attention has been devoted to the frequency of severe and fatal CM incidents. While available data (table 16) diverge greatly, taken together, they suggest that, with the ionic preparations, fatal CM incidents are very rare events, given the frequency of use of these substances.

\begin{tabular}{crlr}
$1:$ & 116,000 & Pendergrass, USA & 1960 \\
\hline $1:$ & 85,000 & Toniolo, Italy & 1966 \\
\hline $1:$ & 61,000 & Wolfromm, France & 1966 \\
\hline $1:$ & 40,000 & Ansell, U.K. & 1970 \\
\hline $1:$ & 54,000 & Fischer, USA & 1972 \\
\hline $1:$ & 30,000 & Witten, USA & 1973 \\
\hline $1:$ & 10,000 & Shehadi, USA & 1975 \\
\hline $1:$ & 20,000 & Shehadi, USA & 1980 \\
\hline $1:$ & 75,000 & Hartmann, USA & 1982
\end{tabular}

Table. 16. Risk of death with i.v. urography 


\section{Nonionic contrast media}

Nonionic contrast media are much better tolerated than ionic contrast media in many ways (table 17). A number of trials investigated the frequency of general reactions after intravenous administration of nonionic X-ray contrast media (table 18), some of them in comparison to conventional ionic X-ray contrast media. The available data allow general statements regarding the frequency of severe reactions but it is not possible to provide sound information on the frequency of deaths related to the use of nonionic contrast media. However, it is very likely that nonionic $\mathrm{X}$-ray contrast media are less frequently related to fatalities or life-threatening conditions (unconsciousness of the patient, conditions requiring an anesthetist etc.) than ionic contrast media (table 18).

On the other hand, it must be borne in mind that all of the known types of side-effects associated with ionic contrast media can also occur during the administration of nonionic contrast media. Only the frequency has actually changed. One must, therefore, always be prepared to treat contrast medium reactions. 


\section{Conventional ionic X-ray-CM \\ Low osmolar ionic X-ray-CM \\ Nonionic \\ X-Ray-CM}

General reactions (nausea, vomiting, allergoid side-effects, some cardiovascular reactions)

\begin{tabular}{llll}
\hline Neural tolerance & $\boldsymbol{\nabla}$ & $\boldsymbol{\Delta}$ \\
\hline $\begin{array}{l}\text { Osmolality-dependent } \\
\text { effects (pain, vasodilation, } \\
\text { bradycardia, diuresis etc.) }\end{array}$ & $\boldsymbol{\nabla}$ & $\boldsymbol{\nabla}$ & $\boldsymbol{\Delta}$ \\
\hline $\begin{array}{l}\text { Calcium binding } \\
\text { (cardiodepression) }\end{array}$ & $\boldsymbol{\nabla}$ & $\bullet$ & $\boldsymbol{\Delta}$ \\
\hline General renal tolerance, i.v. & $\bullet$ & $\bullet$ & $\boldsymbol{\Delta}$ \\
\hline Renal arteriography & $\boldsymbol{\nabla}$ &
\end{tabular}

$\boldsymbol{\nabla}$ poorly tolerated $\Delta$ much better tolerated

no significant difference or difference not proven

Table. 17. Differences in tolerance between the individual types of X-ray contrast media for urography, angiography and $\mathrm{CT}$

\begin{tabular}{|c|c|c|c|c|}
\hline \multirow[b]{2}{*}{ Trial } & \multicolumn{2}{|c|}{ Nonionic CM side-effects } & \multicolumn{2}{|c|}{ Ionic CM side-effects } \\
\hline & total & severe/very severe & total & severe/very severe \\
\hline $\begin{array}{l}\text { Schrott [82] } \\
50,660 \text { cases }\end{array}$ & $2.1 \%$ & $0.01 \%$ & & \\
\hline $\begin{array}{l}\text { Palmer [83] } \\
30,228 \text { nonionic cases } \\
79,278 \text { ionic cases }\end{array}$ & $1.2 \%$ & $0.02 \%$ & $3.8 \%$ & $0.09 \%$ \\
\hline $\begin{array}{l}\text { Katayama [6] } \\
168,363 \text { nonionic cases } \\
169,284 \text { ionic cases }\end{array}$ & $3.1 \%$ & $0.04 \%$ & $12.7 \%$ & $0.22 \%$ \\
\hline $\begin{array}{l}\text { Cochran [84] } \\
73,039 \text { nonionic cases } \\
12,916 \text { inonic cases }\end{array}$ & $0.3 \%$ & $0.02 \%$ & $1.3 \%$ & $0.05 \%$ \\
\hline
\end{tabular}

Table. 18. Frequency of side-effects related to i.v. injection of nonionic and ionic X-ray contrast media (severe/very severe reaction requires that the patient be immediately hospitalized)

\section{Delayed reactions}

Following the introduction of nonionic contrast media, reactions have been reported which are first noticed hours or even days after administration.

A comparative study of iohexol and iopamidol was performed in 2,382 patients undergoing CT examinations.
Reactions delayed by up to 2 days were twice as common as reactions occurring in the first 30 minutes. Headache and rash were the most frequent delayed reactions. Although all delayed reactions were mild in intensity, the attending physician should inform the patient about the possibility of delayed reactions [85). 
A greater frequency of allergy-like delayed reactions was observed for intravascular administration of nonionic dimeric X-ray contrast media. These also included rare severe events lasting for several days and requiring therapy. The causes, risk factors, mechanisms of action, and the actual frequency especially of severe delayed hypersensitivity reactions remain unknown despite concerted efforts to elucidate them [86].

\section{Causes of contrast media reactions}

Contrast medium side-effects generally cannot be explained by a single mechanism [87]. The type of contrast agent and its particular pharmacological properties, the examination technique employed, such as the magnitude of the dose and the mode of administration, and finally, the patient's attitude, such as fear, are all regarded as important contributing factors. CM side effects can be classified in several ways, but the most appropriate is by their cause.

Classification by cause:

- General or largely dose-independent CM reactions (anaphylaxis).

- Chemotoxic, local and cardiovascular effects, dose-dependent side-effects (concentration. volume).

\section{General reactions (anaphylactoid reactions)}

General or largely dose-independent CM reactions range from mild reactions, such as urticaria, to moderately severe reactions, such as bronchospasm, and severe reactions, such as collapse or even cardiac arrest, to fatal outcome. Severe and fatal reactions are rare. The mortality reported for conventional ionic CM is one out of 10,000 to 100,000 patients. 
These reactions may occur in any patient without any warning. Because of this, the attending physician must be prepared for all emergency measures, ranging from medications to artificial respiration.

General CM reactions have little or nothing to do with the osmolality of the CM. They can occur after administration of very small amounts of diluted and even isotonic $\mathrm{CM}$.

They are observed most frequently after i.v. administration, but also in arteriography and other examination procedures.

General CM reactions have been attributed to the following underlying mechanisms:

- Effects on plasma protein, the complement system, blood coagulation, and/or vascular endothelium

- Effects mediated by the central nervous system

- Cross-reaction of the CM with antibodies which were not, however, originally formed against the CM.

\section{Prophylaxis of general reactions}

It is now generally accepted that nonionic CM cause such reactions considerably less frequently than ionic CM and, in particular, less frequently than the low-osmolar, ionic compound meglumine sodium ioxaglate $[88,89]$. 
Even patients who have exhibited repeat and predictable reactions to the injection of ionic $\mathrm{CM}$ often tolerate nonionic CM without symptoms [90]. Prophylactic effects regarding general $\mathrm{CM}$ reactions to both conventional ionic and nonionic preparations have been demonstrated for certain therapeutic regimes, e.g., oral administration of $32 \mathrm{mg}$ methylprednisolone ( ${ }^{*} 2$ tablets methylprednisolone, 16 mg) 6-12 and 2 hours before the examination or the combined administration of $\mathrm{H} 1{ }^{* *} \mathrm{H} 1$ blocker: dimethindene maleate; intravenous injection of $0.2 \mathrm{mg} / \mathrm{kg} \mathrm{BW}$ ) and $\mathrm{H} 2$ blockers $\left({ }^{* *} \mathrm{H} 2\right.$ blocker: cimetidine; intravenous injection of $5 \mathrm{mg} / \mathrm{kg} \mathrm{BW}$ ) [91, 92, 93]. Prophylaxis aims at reducing the frequency of anaphylactoid reactions; however, it is not possible to fully eliminate such reactions by the suggested measures. It can also not be expected that the frequency or severity of any reactions other than anaphylactoid side effects (e.g., neural, renal, most cardiovascular reactions or pain) will be reduced by prophylactic treatment of patients with corticoids or histamine receptor blockers before $\mathrm{CM}$ administration.

In patients at risk (patients with allergic and cardiopulmonary conditions), prophylaxis with $\mathrm{H} 1$ and $\mathrm{H} 2$ blockers reduces the frequency of side-effects, even when nonionic X-ray contrast media are administered, while patients without risk factors have no benefits from $\mathrm{H} 1$ and $\mathrm{H} 2$ blockers [94]. What is certain is that calm and assured management of patients and distraction of their attention during the examination can help to avoid side effects. 


\section{Dose-dependent side effects}

Distinctly dose-dependent side effects include sensations of pain and heat, some circulatory disturbances, and renal impairment. These adverse effects are attributable to the osmolality and pharmacological properties of the agent, and it is not always possible to differentiate between these two components. All nonionic CM have markedly lower osmolality than conventional ionic contrast agents, which is one reason why they have replaced ionic contrast media to a significant degree.

The advantages of nonionic CM are especially obvious in:

- Pain-intensive applications, since osmolality is the main factor determining the painfulness of a contrast medium.

- Angiographic examinations with high total dosages from a number of single injections, e.g., cardioangiography, femoral arteriography, conventional angiography of several vascular regions, i.v. DSA, and angioplasty.

The kidney, which eliminates the CM for several hours after the examination, is regarded as the critical organ in such examinations. Effects of the nonionic CM Ultravist on renal function were examined in various clinical trials. Renal function was not recognizably impaired in any of the patients examined. The cardiovascular effects of conventional ionic $\mathrm{CM}$ are largely attributable to their high osmolality:

- Vasodilatation, reduction of peripheral resistance, and a decrease of blood pressure.

- Hypervolemia.

- Bradycardia in cardioangiography.

Nonionic CM have less of an influence on the heart and circulation. 


\section{Prophylaxis and therapy of severe iodine-induced hyperthyroidism}

When iodinated CM are administered, a healthy thyroid gland can adjust to an iodide surplus in a host of ways without increasing hormone production.

In a diseased thyroid, these selfregulating mechanisms can fail to function. In this way, a diagnostic or therapeutic iodine administration can result in severe metabolic dysfunctions such as decompensated hyperthyroidism and thyrotoxic crisis. A special risk exists for goiter patients (struma) and patients with hyperthyroidism in general. For this reason, iodinated CM should not be administered before hyperthyroidism has been ruled out. According to our present state of knowledge, the risk that hyperthyroidism will develop is not diminished by nonionic CM such as Ultravist.

The risk of hyperthyroidism is exclusively determined by the iodine and/or iodide contents of the $\mathrm{CM}$. Osmolality and possible chemically toxic properties do not play any role. The renal, water-soluble, ionic and nonionic $\mathrm{X}$-ray $\mathrm{CM}$ - as a result of the way they are produced - contain only traces of iodide, and very little of the stable chemically bonded iodine is released in the organism. Nevertheless, even these small amounts can result in further functional deterioration of a severely damaged thyroid gland (e.g., with manifest hyperthyroidism).

A peculiarity of iodine-induced hyperthyroidism is that deterioration customarily does not occur immediately but weeks or months after iodine administration. 
If diagnostic measures make it essential to use iodinated CM in a patient with hyperthyroidism, combined perchlorate and carbimazole (or thiamizole) should be given for prophylaxis. Administration of perchlorate $(3 \times 300 \mathrm{mg}$ daily, from 1-2 days before until 1 week after CM administration) inhibits the absorption of iodide by the thyroid gland; administration of methimazole simultaneously blocks hormone synthesis $(2 \times 20 \mathrm{mg}$ thiamizole daily, from 1-2 days before until 2-3 weeks after CM administration).

The longer retention times of biliary CM require a further 3 weeks of treatment with half the dose of thiamizole (carbimazole) [95].

\section{Renal damage}

Ionic and nonionic contrast media used in urography, angiography and computed tomography (CT) are eliminated mainly via the kidneys. Isolated cases of reduced kidney function including acute renal failure following intravenous and intra-arterial administration have been described in the literature.

However, the risks related to intravenous administration in patients without obvious risk factors has been, to some extent, exaggerated in the past. Systematic comparative studies [96, 97] showed that spontaneous changes in kidney function in patients who had received no contrast media were not significantly less common than in patients receiving contrast media, e.g., for CT.

\section{Renal insufficiency}

When ionic CM is administered intravenously for urography in patients with healthy kidneys, deterioration in renal function is hardly ever observed; it seems to be, however, somewhat more frequent in those with renal insufficiency. 
Deterioration is defined as an increase of $1 \mathrm{mg} / \mathrm{dl}$ in serum creatinine. Nonionic CM are well tolerated by the kidneys of patients with reduced renal function - e.g., after administering iopromide intravenously - and several trials identified no clinically relevant changes in the parameters describing renal tolerance: serum creatinine, creatinine clearance, and urinary protein. Well-designed clinical studies demonstrated that natural fluctuations of serum creatinine occur to the same extent in patients who received no CM [98, 99, 100].

In arteriography, part of the CM can flow directly into the kidneys in almost undiluted form. Under these conditions, impairment of renal function is more likely, even though nonionic CM have been better tolerated, especially in renal arteriography, than ionic CM [101].

\section{Plasmocytoma}

Disturbed renal function is found in a high percentage of patients with multiple myeloma. Isolated cases of acute renal failure in myeloma patients following urography have been reported in the literature. Almost all of these patients presented additional risk factors prior to $\mathrm{CM}$ administration, such as severe dehydration and/or sepsis. In addition, almost all of these urographic examinations were performed decades ago with diiodinated CM. In more recent retrospective studies of patients with confirmed multiple myeloma who underwent intravenous urography, no temporal connection could be established between deterioration of renal function and CM administration. In the opinion of experts, given adequate hydration and the use of modern CM, plasmocytoma is no a priori contraindication for $\mathrm{CM}$ administration. 
Rather, more recent studies suggest that the principal risk factors for acute renal damage in patients with multiple myeloma are hypercalcemia, dehydration, infections, and Bence Jones proteinuria [102]. Patients with serum creatinine levels above $2 \mathrm{mg} \%$ should not undergo urography.

\section{Diabetes mellitus}

Proteinuria and elevated serum creatinine in patients with insulin-dependent diabetes mellitus indicate advanced diabetic glomerular sclerosis. These patients are more likely to show deterioration in renal function after $\mathrm{CM}$ administration. As a rule, only severe proteinuria $(300 \mathrm{mg} / 24 \mathrm{~h})$ will significantly increase the risk of acute renal failure in a diabetes mellitus patient. In this condition, high concentrations of dissolved materials contained in urine tend to precipitate in the presence of $\mathrm{CM}$ and may cause tubular blockage.

\section{Prophylaxis}

Patients with risk factors for reduced renal tolerance should not receive high intravascular doses of contrast media. These risk factors include

- limited renal function in conjunction with

- a long history of insulin-dependent diabetes mellitus,

- cardiac insufficiency,

- and advanced age.

Several alternative imaging modalities including ultrasound, magnetic resonance imaging, and arterial digital subtraction angiography are available to avoid or diminish the use of X-ray contrast media in these patients. 
The following prophylactic measures are recommended to reduce the risk of renal damage [103]:

- ensure adequate hydration by sufficient water intake

- avoid multiple examinations with X-ray contrast media during a short period of only a few days

- discontinue medications which might impair renal function

A recommendation for medical prophylaxis adequately confirmed by clinical studies cannot at present be made. A possible prophylactic effect is known primarily for theophylline. However, the optimal dose for prevention of CIN has not been established [104].

\section{Pregnant patients}

The use of CM during pregnancy has not yet been proven to be harmless although toxicological studies in animals and clinical experience do not indicate any particular risk. Since exposure to radiation should be avoided during this period, the potential risks of any X-ray examination must be given careful consideration.

\section{Breast-feeding mothers}

Only very small proportions of renal $\mathrm{CM}$ and i.v. cholegraphic agents enter breast milk. They are barely reabsorbed in breast-feeding so that one can assume that there is no danger to the infant. Even with oral cholecystographic agents, exposure of the infant is minimal. 


\section{Pheochromocytoma}

Patients with pheochromocytoma often undergo $X$-ray examinations prior to surgery to determine the site, size, and number of tumors. Such examinations are not totally without risk to patients. In angiography, especially with selective CM injection into tumor vessels, a spontaneous release of massive amounts of catecholamine into the blood stream can occur, a reaction known from other situations of stress.

This results in a hypertensive crisis. A critical drop in blood pressure is a much more unusual, but more serious incident. Angiographic procedures requiring selective $\mathrm{CM}$ administration should only be performed in pheochromocytoma patients after adequate treatment with alpha-adrenergic receptor blockers for a sufficient length of time (10-14 days).

\section{Sickle-cell anemia}

In sickle-cell anemia patients, some erythrocytes contain the abnormal hemoglobin $\mathrm{S}$. If $\mathrm{O}_{2}$ pressure is lowered or blood osmolality is raised, HbS-containing blood corpuscles become inelastic and stretched. These erythrocytes can no longer pass through capillaries as easily, which can lead to stasis and to different forms of organ damage.

Administering high-osmolar CM to such patients is not without problems. The risk is highest for intra-arterial $\mathrm{CM}$ injection, especially in high doses, as is the case in cerebral and cardiac arteriography. In vitro studies comparing the effect of ionic and nonionic CM in terms of sickle-shaped changes of erythrocytes reveal that these changes are significantly less pronounced when nonionic CM is used [105]. 
Open Access This chapter is licensed under the terms of the Creative Commons Attribution 4.0 International License (http://creativecommons.org/licenses/by/4.0/), which permits use, sharing, adaptation, distribution and reproduction in any medium or format, as long as you give appropriate credit to the original author(s) and the source, provide a link to the Creative Commons license and indicate if changes were made.

The images or other third party material in this chapter are included in the chapter's Creative Commons license, unless indicated otherwise in a credit line to the material. If material is not included in the chapter's Creative Commons license and your intended use is not permitted by statutory regulation or exceeds the permitted use, you will need to obtain permission directly from the copyright holder. 